\title{
Complex entropy and resultant information measures
}

\author{
Roman F. Nalewajski ${ }^{1}$
}

Received: 30 March 2016 / Accepted: 25 May 2016 / Published online: 6 June 2016

(C) The Author(s) 2016. This article is published with open access at Springerlink.com

Abstract Classical and nonclassical contributions to Author's resultant Shannon- and Fisher-type measures of the information content in general electronic state $\varphi(\boldsymbol{r})=$ $R(\boldsymbol{r}) \exp [\mathrm{i} \phi(\boldsymbol{r})]$, due to the state probability density $p(\boldsymbol{r})=R(\boldsymbol{r})^{2}$ and its phase $\phi(\boldsymbol{r})$ or current $\boldsymbol{j}(\boldsymbol{r})=(\hbar / m) p(\boldsymbol{r}) \nabla \phi(\boldsymbol{r})$ distributions, respectively, are reexamined. The components of the overall entropy,

$$
S[\varphi] \equiv-\int p(\boldsymbol{r})[\ln p(\boldsymbol{r})+2 \phi(\boldsymbol{r})] d \boldsymbol{r} \equiv S[p]+S[\phi],
$$

are shown to determine the real and imaginary parts of the state complex Shannon entropy,

$$
H[\varphi] \equiv-2\langle\varphi|\ln \varphi| \varphi\rangle=S[p]+\mathrm{i} S[\phi],
$$

a natural quantum-amplitude generalization of the classical Shannon entropy. Its contributions are related to the associated terms in the state resultant Fisher information,

$$
\begin{aligned}
I[\varphi] & \equiv-4\left\langle\varphi\left|\nabla^{2}\right| \varphi\right\rangle \equiv \int p(\boldsymbol{r})\left\{[\nabla \ln p(\boldsymbol{r})]^{2}+[2 \nabla \phi(\boldsymbol{r})]^{2}\right\} d \boldsymbol{r} \equiv I[p]+I[\phi] \\
& =I[p]+\int p(\boldsymbol{r})[(2 m / \hbar) \boldsymbol{j}(\boldsymbol{r}) / p(\boldsymbol{r})]^{2} d \boldsymbol{r} \equiv I[p]+I[\boldsymbol{j}],
\end{aligned}
$$

$凶$ Roman F. Nalewajski

nalewajs@chemia.uj.edu.pl

1 Department of Theoretical Chemistry, Jagiellonian University, R. Ingardena 3, 30-060 Cracow, Poland 
and the gradient entropy:

$$
\tilde{I}[\varphi] \equiv\left\langle\varphi\left|\left[(\nabla \ln p)^{2}+(\mathrm{i} 2 \nabla \phi)^{2}\right]\right| \varphi\right\rangle=I[p]-I[\phi]=\tilde{I}[p]+\tilde{I}[\phi]
$$

Keywords Complex entropy · Fisher information · Information theory · Nonclassical information · Resultant information measures · Shannon entropy

\section{Introduction}

The crucial problem in entropic theories of molecular electronic structure is the quantum generalization of the classical entropy/information concepts of Fisher [1] and Shannon [2], appropriate for complex amplitudes (wavefunctions). Both the particle probability distribution and its phase or current densities ultimately contribute to the resultant information descriptors of molecular systems. Such resultant measures of the information content in electronic states, combining the classical contributions due to wavefunction modulus and their nonclassical supplements due to state phase, have been recently proposed, e.g., [3-8]. The electron density generates only the classical part of the overall information content, while the wavefunction phase or its gradient (probability-current) generate its nonclassical complement in the associated resultant measure. The quantum extension of the classical Fisher (gradient) information has been proposed using its association with the average kinetic energy of electrons and the corresponding resultant global entropy has been subsequently inferred using the relation between densities of the classical Fisher and Shannon measures [3-8]. Although, for simplicity, in what follows we assume the one-electron case the modulus (density) and phase (current) aspects of general electronic states can be separated using the Harriman [9], Zumbach and Maschke [10] construction of Slater determinants yielding the specified electron density [4,11].

In the present communication we relate the classical and nonclassical components of such generalized entropy/information descriptors of molecular states to the real and imaginary parts of the complex entropy concept, a natural extension of the classical Shannon entropy, provided by the functional of the system complex electronic wavefunction (quantum probability amplitude).

\section{Information components}

The average Fisher [1] measure of the gradient-information content in the probability density $p(\boldsymbol{r})=|\varphi(\boldsymbol{r})|^{2}=R(\boldsymbol{r})^{2}$ of the single-particle state $|\varphi\rangle$ described by the wavefunction $\varphi(\boldsymbol{r})=\langle\boldsymbol{r} \mid \varphi\rangle=R(\boldsymbol{r}) \exp [\mathrm{i} \phi(\boldsymbol{r})]$ is reminiscent of von Weizsäcker's [12] inhomogeneity correction to the kinetic energy functional,

$$
\begin{aligned}
I[p] & =\int[\nabla p(\boldsymbol{r})]^{2} / p(\boldsymbol{r}) d \boldsymbol{r}=\int p(\boldsymbol{r})[\nabla \ln p(\boldsymbol{r})]^{2} d \boldsymbol{r} \equiv \int p(\boldsymbol{r}) I_{p}(\boldsymbol{r}) d \boldsymbol{r} \\
& =4 \int[\nabla R(\boldsymbol{r})]^{2} d \boldsymbol{r} \equiv I[R] .
\end{aligned}
$$


Its amplitude form $I[R]$ reveals that it measures the average length of the modulusgradient. This classical descriptor characterizes an effective compactness ("narrowness") of the particle position distribution. The complementary descriptor of the classical Shannon [2] entropy,

$$
S[p]=-\int p(\boldsymbol{r}) \ln p(\boldsymbol{r}) d \boldsymbol{r} \equiv \int p(\boldsymbol{r}) S_{p}(\boldsymbol{r}) d \boldsymbol{r} \equiv-2 \int R^{2}(\boldsymbol{r}) \ln R(\boldsymbol{r}) d \boldsymbol{r} \equiv S[R],
$$

reflects the average indeterminacy ("spread") of this random variable. It provides the amount of information received, when this uncertainty is removed by an appropriate localization experiment: $I^{\mathrm{S}}[p] \equiv S[p]$. The densities-per-electron of these probability/modulus functionals are seen to satisfy the classical (nonlinear) relation

$$
I_{p}(\boldsymbol{r})=\left[\nabla S_{p}(\boldsymbol{r})\right]^{2}
$$

The resultant entropy/information descriptors of state $|\varphi\rangle$ combine these familiar classical contributions and the associated nonclassical supplements due to the state spatial phase or probability current [3-8]:

$$
\begin{aligned}
I[\varphi] & =-4\left\langle\varphi\left|\nabla^{2}\right| \varphi\right\rangle \equiv\langle\varphi|\hat{\mathbf{I}}| \varphi\rangle=4 \int|\nabla \varphi(\boldsymbol{r})|^{2} d \boldsymbol{r} \equiv \int p(\boldsymbol{r}) I(\boldsymbol{r}) d \boldsymbol{r} \\
& =I[p]+4 \int p(\boldsymbol{r})[\nabla \phi(\boldsymbol{r})]^{2} d \boldsymbol{r} \equiv \int p(\boldsymbol{r})\left[I_{p}(\boldsymbol{r})+I_{\phi}(\boldsymbol{r})\right] d \boldsymbol{r} \equiv I[p]+I[\phi] \\
& =I[p]+\left(\frac{2 m}{\hbar}\right)^{2} \int j^{2}(\boldsymbol{r}) / p(\boldsymbol{r}) d \boldsymbol{r} \\
& =\int p(\boldsymbol{r})\left[I_{p}(\boldsymbol{r})+I_{j}(\boldsymbol{r})\right] d \boldsymbol{r} \equiv I[p]+I[j] \\
S[\varphi] & =-\langle\varphi|\ln p+2 \phi| \varphi\rangle \equiv\langle\varphi|\hat{\mathrm{S}}| \varphi\rangle=\int \varphi(\boldsymbol{r})^{*} \hat{\mathrm{S}}(\boldsymbol{r}) \varphi(\boldsymbol{r}) d \boldsymbol{r} \\
& =\int p(\boldsymbol{r})\left[S_{p}(\boldsymbol{r})-2 \phi(\boldsymbol{r})\right] d \boldsymbol{r} \equiv \int p(\boldsymbol{r})\left[S_{p}(\boldsymbol{r})+S_{\phi}(\boldsymbol{r})\right] d \boldsymbol{r} \\
& =\int p(\boldsymbol{r}) S(\boldsymbol{r}) d \boldsymbol{r} \equiv S[p]+S[\phi], \\
\tilde{I}[\varphi] & =I[p]-I[\phi] \equiv \tilde{I}[p]+\tilde{I}[\phi]=\int p(\boldsymbol{r})\left[\tilde{I}_{p}(\boldsymbol{r})+\tilde{I}_{\phi}(\boldsymbol{r})\right] d \boldsymbol{r} \\
& \equiv \int p(\boldsymbol{r}) \tilde{I}(\boldsymbol{r}) d \boldsymbol{r} .
\end{aligned}
$$

Above, these information components have been expressed as expectation values of the related real (multiplicative) "operators" in position representation, measuring the resultant densities-per-electron of the gradient information and entropy,

$$
I(\boldsymbol{r})=[\nabla \ln p(\boldsymbol{r})]^{2}+4[\nabla \phi(\boldsymbol{r})]^{2} \text { and } \tilde{I}(\boldsymbol{r})=[\nabla \ln p(\boldsymbol{r})]^{2}-4[\nabla \phi(\boldsymbol{r})]^{2} \text {, }
$$


and of the overall entropy:

$$
S(\boldsymbol{r})=-[\ln p(\boldsymbol{r})+2 \phi(\boldsymbol{r})] .
$$

The generalized gradient-information of Eq. (4), the expectation value of quantummechanical operator $\hat{\mathrm{I}}=-4 \nabla^{2}=\left(8 \mathrm{~m} / \hbar^{2}\right) \hat{\mathrm{T}}$, is proportional to the average kinetic energy $T[\varphi]=\langle\varphi|\hat{\mathrm{T}}| \varphi\rangle$ corresponding to the Hermitian operator $\hat{\mathrm{T}}(\boldsymbol{r})=-\left[\hbar^{2} /(2 m)\right] \Delta$,

$$
I[\varphi]=\left(8 m / \hbar^{2}\right) T[\varphi],
$$

and reflects the state gradient-deterministic aspect. The nonclassical densities of the resultant gradient information [Eq. (4)] and global entropy [Eq. (5)], respectively, obey the classical relation of Eq. (3):

$$
I_{\phi}(\boldsymbol{r})=\left[\nabla S_{\phi}(\boldsymbol{r})\right]^{2} .
$$

One observes, however, that in the resultant gradient-entropy [Eq. (6)], which describes the state gradient-indeterminicity facet, the nonclassical contribution changes sign, $\tilde{I}[\phi]=-I[\phi]$, so that the nonclassical Shannon and Fisher entropy densities satisfy the modified relation:

$$
\tilde{I}_{\phi}(\boldsymbol{r})=-\left[\nabla S_{\phi}(\boldsymbol{r})\right]^{2}
$$

\section{Complex entropy concept}

Thus, the resultant entropy/information functionals combine the classical and nonclassical contributions, which separately obey the nonlinear relation of Eqs. (3) and (8). However, the nonlinear character of the mutual dependencies between these Shannon and Fisher components precludes this relation to be also satisfied by the resultant densities themselves, unless the two Shannon components are regarded as components of the vector entity $\vec{S}(\boldsymbol{r}) \equiv \boldsymbol{e}_{p} S_{p}(\boldsymbol{r})+\boldsymbol{e}_{\phi} S_{\phi}(\boldsymbol{r})$, in the geometrical framework of the probability and phase degrees-of-freedom then determining the independent (orthogonal) directions represented by the perpendicular unit vectors $\boldsymbol{e}_{p}$ and $\boldsymbol{e}_{\phi}, \boldsymbol{e}_{p} \cdot \boldsymbol{e}_{\phi}=0$ [3],

$$
I(\boldsymbol{r})=I_{p}(\boldsymbol{r})+I_{\phi}(\boldsymbol{r})=[\nabla \cdot \vec{S}(\boldsymbol{r})]^{2}=\left[\nabla S_{p}(\boldsymbol{r})\right]^{2}+\left[\nabla S_{\phi}(\boldsymbol{r})\right]^{2}
$$

However, this vector interpretation does not justify the change of sign in Eq. (9). The proper explanation calls for the density of the complex entropy concept, defined in an alternative vector framework of the complex plane,

$$
H(\boldsymbol{r})=S_{p}(\boldsymbol{r})+\mathrm{i} S_{\phi}(\boldsymbol{r}) \equiv=-[\ln p(\boldsymbol{r})+2 \mathrm{i} \phi(\boldsymbol{r})],
$$

with the Shannon phase-entropy component $S_{\phi}(\boldsymbol{r})$ being then attributed to the imaginary part. This generalized entropy indeed naturally follows from the classical entity, 
when one refers to the multi-valued logarithmic function of the complex argument $z=|z| \exp (\mathrm{i} \alpha)$

$$
\operatorname{Ln} z=\ln |z|+\mathrm{i}(\alpha+2 \pi k), \quad k=0, \pm 1, \pm 2, \ldots,
$$

or its single-valued branch $k=0$ :

$$
\ln z=\ln |z|+\mathrm{i} \alpha
$$

Indeed, the resultant entropy of Eq. (5) then reflects the expectation value of the complex (multiplicative) logarithmic operator of Eq. (11) expressed in terms of the electronic state $\varphi$ itself,

$$
\begin{aligned}
\hat{\mathrm{H}}(\boldsymbol{r}) & =H(\boldsymbol{r})=-2 \ln \varphi(\boldsymbol{r})=-[\ln p(\boldsymbol{r})+2 \mathrm{i} \phi(\boldsymbol{r})] \equiv H_{p}(\boldsymbol{r})+H_{\phi}(\boldsymbol{r}), \\
H[\varphi] & =\langle\varphi|-2 \ln \varphi| \varphi\rangle \equiv\langle\varphi|\hat{\mathrm{H}}| \varphi\rangle=S[p]+\mathrm{i} S[\phi] \\
& =S[R]+\mathrm{i} S[\phi] \equiv H[p]+H[\phi]
\end{aligned}
$$

The complex entropy thus provides a natural complex-amplitude generalization of the the familiar classical measure of the entropy content in probability distribution. The non-Hermitian entropy operator $\hat{\mathrm{H}}=-2 \ln \varphi$ [Eq. (14)] then generates the probability and phase Shannon-type contributions as the real and imaginary parts of the resultant complex entropy.

Thus, the Hermitian operator $\hat{\mathrm{I}}=-4 \nabla^{2}=\left(8 \mathrm{~m} / \hbar^{2}\right) \hat{\mathrm{T}}$ gives rise to the real expectation value of the state resultant information content $I[\varphi]$, while the non-Hermitian entropy operator $\hat{H}(\boldsymbol{r})=-2 \ln \varphi(\boldsymbol{r})$ generates the state complex average quantity $H[\varphi]$. The gradient analogs in Eq. (6) then follow from the same type of a mutual relation [Eqs. (3) and (8)] between the information and entropy densities [compare Eq. (9)]:

$$
\tilde{I}_{p}(\boldsymbol{r})=\left[\nabla H_{p}(\boldsymbol{r})\right]^{2} \text { and } \tilde{I}_{\phi}(\boldsymbol{r})=\left[\nabla H_{\phi}(\boldsymbol{r})\right]^{2}=\left[\mathrm{i} \nabla S_{\phi}(\boldsymbol{r})\right]^{2}=-\left[\nabla S_{\phi}(\boldsymbol{r})\right]^{2} .
$$

Open Access This article is distributed under the terms of the Creative Commons Attribution 4.0 International License (http://creativecommons.org/licenses/by/4.0/), which permits unrestricted use, distribution, and reproduction in any medium, provided you give appropriate credit to the original author(s) and the source, provide a link to the Creative Commons license, and indicate if changes were made.

\section{References}

1. R.A. Fisher, Proc. Camb. Philos. Soc. 22, 700 (1925)

2. C.E. Shannon, Bell Syst. Tech. J. 27, 379 (1948)

3. R.F. Nalewajski, Quantum Information Theory of Molecular States (Nova Science, New York, 2016)

4. R.F. Nalewajski, Ann. Phys. (Leipzig) 525, 256 (2013)

5. R.F. Nalewajski, J. Math. Chem. 52, 588 (2014)

6. R.F. Nalewajski, Mol. Phys. 112, 2587 (2014)

7. R.F. Nalewajski, Int. J. Quantum Chem. 115, 1274 (2015) 
8. R.F. Nalewajski, J. Math. Chem. 53, 1126 (2015)

9. J.E. Harriman, Phys. Rev. A 24, 680 (1981)

10. G. Zumbach, K. Maschke, Phys. Rev. A 28, 544 (1983); Erratum, Phys. Rev. A 29, 1585 (1984)

11. R.F. Nalewajski, J. Math. Chem. 51, 369 (2013)

12. C.F. von Weizsäcker, Z. Phys. 96, 431 (1935) 